\title{
Abortursachen beim Pferd - eine Übersicht der Literatur und eigene Auswertungen
}

\author{
Ramona Weber'; Rainer Hospes'; Axel Wehrend ${ }^{2}$ \\ ${ }^{1}$ Tierarztpraxis Wessling, Siegen; ${ }^{2}$ Klinik für Geburtshilfe, Gynäkologie und Andrologie der Groß- und Kleintiere mit Tierärztlicher Ambulanz, \\ Justus-Liebig-Universität Gießen
}

\section{Schlüsselwörter \\ Stute, Abort, Vollblutzucht, Infektion}

\section{Zusammenfassung}

Ziel dieser Arbeit war, den aktuellen Stand der Literatur über mögliche Ursachen, die klinische Symptomatik und Pathogenese des Aborts bei der Stute zusammenzufassen und durch Auswertung von Patientendaten die Häufigkeit verschiedener Abortursachen in der deutschen Vollblutzucht darzustellen. Hierzu wurde eine Literaturauswertung unter Verwendung von Fachdatenbanken, veterinärmedizinischen Fachzeitschriften und Lehrbüchern vorgenommen und zusätzlich die Untersuchungsergebnisse von 123 Aborten des Vollblutzuchtgebiets Deutschland Mitte ausgewertet. Die in der Literatur am häufigsten beschriebene Abortursache ist die bakterielle Infektion. Große Bedeutung haben auch Aborte durch equines Herpesvirus (EHV) 1/4, Zwillingsgraviditäten und Nabelstranganomalien. Beispiele seltenerer Abortursachen sind equine virale Arteritis (EVA), infektiöse Anämie der Einhufer (EIA), Infektionen durch Pilze oder Protozoen, fetale Missbildungen und Erkrankungen der Mutterstute (Begleitabort). In der eigenen Studie konnte in $47,2 \%$ der Fälle keine Abortursache gefunden werden. Ein Infektionsgeschehen lag bei $26,8 \%$ der Aborte vor. Infektionserreger waren in $17,1 \%$ der Fälle Bakterien, in $8,9 \%$ Viren (EHV 1/4) und in $0,8 \%$ Pilze. Als Hauptursache für einen nichtinfektiösen Abort erwies sich die Zwillingsgravidität $(21,1 \%)$. Andere Gründe nichtinfektiöser Aborte waren fetale Missbildung (3,3\%), Nabelstrangulation $(0,8 \%)$ und Uterustorsion (0,8\%). Die Ergebnisse der Studie legen nahe, dass in Zukunft ein größeres Augenmerk auf sonographische Untersuchungen in der Frühträchtigkeit zum Ausschluss einer Zwillingsgravidität gelegt werden sollte, um die Abortrate weiter zu senken. Um die Aufklärungsrate bezüglich der Abortursachen zu erhöhen, könnte es helfen, bei der Untersuchung des Abortmaterials auch seltenere Abortursachen (z B. EVA oder Chlamydien) routinemäßig labordiagnostisch abzuklären.

\section{Keywords}

Mare, abortion, thoroughbred breeding, infection

\section{Summary}

The aim of this study was to summarize the current status of the possible causes, clinical symptoms and pathogenesis of abortion in the mare and to evaluate the frequency of varying causes of abortion in German thoroughbred breeding. An analysis of the literature using electronic libraries, journals and textbooks was performed. In addition, the results of examinations of 123 abortions of thoroughbred breeding in central Germany were evaluated. In the literature, bacterial infections are the most frequently described cause of abortion. Other important causes are infections with equine herpesvirus (EHV)-1 or -4 , twin pregnancy and umbilical cord anomalies. Examples of rare causes of abortion are equine viral arteritis (EVA), equine infectious anemia, fungal or parasitic infections, fetal malformations and diseases of the mare. In our study of 123 abortions, no cause was found in $47.2 \%$ of the analyzed abortions. Infections were responsible for $26.8 \%$ of the abortions. The main pathogens were bacteria in $17.1 \%$ of the cases followed by viruses (EHV-1/4) in $8.9 \%$ of the cases. Fungal infection was diagnosed in $0.8 \%$ of the cases. The most common cause of a non-infectious abortion was twin pregnancy $(21.1 \%)$. Other causes of non-infectious abortion were fetal malformations (3.3\%), umbilical cord strangulations $(0.8 \%)$ and uterine torsion $(0.8 \%)$. The results of our own study suggest that in the future greater attention should be paid to ultrasonographic examinations in the early pregnancy to reduce the rate of abortions because of a twin pregnancy. To increase the detection rate of unknown causes of abortion, rarer causes of abortion should be investigated, including for EVA and chlamydia, as a matter of routine.
Korrespondenzadresse

Prof. Dr. A. Wehrend

Klinik für Geburtshilfe, Gynäkologie und Andrologie

der Groß- und Kleintiere mit Tierärztlicher Ambulanz

der Universität Gießen

Frankfurter Straße 106

35392 Gießen

E-Mail:Axel.Wehrend@vetmed.uni-giessen.de
Causes of abortion in horses - overview of the literature and own evaluations

Tierarztl Prax Ausg G Grosstiere Nutztiere 2018; 46: 35-42 http://dx.doi.org/10.15653/TPG-170517

Eingegangen: 25. August 2017

Akzeptiert nach Revision: 12. November 2017 


\section{Einleitung}

Unter einem Abort wird der Abbruch einer Trächtigkeit verstanden, bevor der Fetus extrauterin lebensfähig ist. Davon abzugrenzen sind der embryonale Fruchttod, der als Verlust des Embryos im ersten Trächtigkeitsmonat definiert wird, und die Frühgeburt, die die Geburt eines lebensfähigen, aber unreifen Fohlens bezeichnet. Bei einer Totgeburt handelt es sich um die Geburt eines reifen, jedoch intrauterin abgestorbenen Fohlens zum Zeitpunkt der Geburt (52).

Hinsichtlich des Gesamtzuchtgeschehens auf Verbandsebene ist der Abort beim Pferd nach wie vor von züchterischer wie auch wirtschaftlicher Bedeutung: Die Abortrate in der deutschen Warm- und Vollblutzucht beträgt etwa $4-5 \%(33,45)$ und liegt damit am unteren Rand der in der Literatur (5) allgemein angegebenen Spannweite von 5-15\%. Doch auch im individuellen Einzelfall führt ein Abort, neben der persönlichen Enttäuschung über den Fohlenverlust, zu einem nicht unerheblichen finanziellen Schaden für den Züchter, der sich aus den Tierarztkosten, Deckgebühren und, je nach Zeitpunkt des Ereignisses, dem Verlust einer Zuchtsaison zusammensetzt.

Ziel dieser Übersicht ist, das derzeitige Literaturwissen über den Abort beim Pferd zusammenzufassen und diesen Daten die eigenen Ergebnisse zu Abortursachen bei Vollblutstuten aus einer aktuellen Patientenauswertung gegenüberzustellen.

\section{Literaturübersicht}

\section{Abortursachen}

Die Abortursachen beim Pferd lassen sich in infektiöse und nichtinfektiöse Ursachen einteilen ( $\triangleright$ Tab. 1). Literaturangaben zufolge kann jedoch trotz umfangreicher Untersuchungen in 5-42\% der Fälle keine Abortursache festgestellt werden $(24,33,39,41)$. Die Häufigkeitsangaben über die Ursachen eines Aborts unterscheiden sich je nach Autor und untersuchter Pferdepopulation deutlich. In einer umfangreichen Studie zu Fohlenverlusten in der deutschen Vollblutzucht zwischen 1968 und 1990 waren 25\% der Aborte infektiös bedingt, wobei mehr als $50 \%$ davon eine bakterielle Infektion zugrunde lag. In $42 \%$ der infektiös bedingten Aborte wurde eine EHV1-Infektion nachgewiesen. Der Anteil nichtinfektiös bedingter Aborte lag bei $47 \%$. Neben maternalen Organerkrankungen, Nabeltorsionen und fetalen Missbildungen war mit 39\% die Zwillingsträchtigkeit die dominierende Ursache nichtinfektiöser Aborte. In 28\% der untersuchten Fälle ließ sich keine Abortursache finden (33).

\section{Infektiöse Aborte}

\section{Equine Herpesviren}

Der Haupterreger bei einem durch Herpesviren ausgelösten Abort ist das equine Herpesvirus Typ 1 (EHV1), wobei auch das nahe

\begin{tabular}{|c|c|}
\hline Infektiöse Abortursachen & Nichtinfektiöse Abortursachen \\
\hline \multirow{2}{*}{$\begin{array}{l}\text { Viren } \\
\text { - equines Herpesvirus } 1 / 4 \\
\text { - equines Arteritisvirus } \\
\text { - Virus der equinen infektiösen Anämie }\end{array}$} & Mehrlingsträchtigkeit \\
\hline & $\begin{array}{l}\text { Nabelstranganomalien } \\
\text { - Torsion } \\
\text { - Neoplasie (Angiom) }\end{array}$ \\
\hline $\begin{array}{l}\text { Bakterien } \\
\text { - Streptococcus equi subsp. zooepidemicus } \\
\text { - Pseudomonas aeruginosa } \\
\text { - Klebsiella pneumoniae } \\
\text { - Staphylococcus sp. } \\
\text { - E. coli } \\
\text { - Chlamydia sp. } \\
\text { - Leptospira sp. } \\
\text { - Salmonella sp. }\end{array}$ & $\begin{array}{l}\text { Pathologische Veränderungen der Plazenta } \\
\text { - ischämische Nekrose } \\
\text { - Korpusgravidität } \\
\text { - Eihautwassersucht } \\
\text { - Amnionruptur }\end{array}$ \\
\hline $\begin{array}{l}\text { Pilze } \\
\text { - Aspergillus fumigatus } \\
\text { - Mucor sp. } \\
\text { - Candida sp. }\end{array}$ & $\begin{array}{l}\text { Erkrankungen der Stute } \\
\text { Allgemeinerkrankungen (Kolik, Fieber, } \\
\text { Neoplasie) } \\
\text { Uteruserkrankungen (Endometrose, Zervix- } \\
\text { insuffizienz, Narben/Traumata, Fehlbildungen) }\end{array}$ \\
\hline \multirow[t]{2}{*}{$\begin{array}{l}\text { Protozoen } \\
\text { - Theileria equi } \\
\text { - Babesia caballi } \\
\text { - Encephalitozoon cuniculi } \\
\text { - Neospora sp. }\end{array}$} & $\begin{array}{l}\text { Pathologische Veränderungen des Fetus } \\
\text { - chromosomale Veränderungen } \\
\text { - Schilddrüsenerkrankungen } \\
\text { - Fehlbildungen } \\
\text { - Neoplasien }\end{array}$ \\
\hline & $\begin{array}{l}\text { Umweltfaktoren } \\
\text { - Wollraupenspinner (USA, Australien) } \\
\text { - Toxikosen }\end{array}$ \\
\hline
\end{tabular}

Tab. 1

Nichtinfektiöse und infektiöse Abortursachen bei der Stute mit einigen wichtigen Erregern (modifiziert nach 51)

Table 1

Causes of infectious and non-infectious abortion in the mare with some important pathogens (modified according to 51). 
verwandte equine Herpesvirus Typ 4 (EHV4) in Einzelfällen nachgewiesen werden kann (3). 95\% der EHV1-bedingten Aborte finden in den letzten 3 Monaten der Trächtigkeit plötzlich und ohne ankündigende klinische Anzeichen statt (27). In der Regel wird der Fetus mit der Nachgeburt ausgestoßen („Abortus completus“) ( $\triangleright$ Abb. 1). Die Infektion kann direkt über Aerosole oder indirekt über Einstreu, Kleidung und Futter erfolgen. Eine besondere Rolle als Infektionsquelle spielt das Abortmaterial. Aufgrund der hohen Kontagiösität kann es zu seuchenhaften Ausbrüchen in einem Bestand kommen. Die Inkubationszeit beträgt zwischen 14 und 120 Tagen. Der Erreger hat eine hohe Affinität zu den Epithelzellen des oberen Respirationstrakts, wo die primäre Virusreplikation stattfindet. Häufig verläuft die Infektion subklinisch, klinische Symptome wie Fieber, Inappetenz und Nasenausfluss sind möglich. Durch die hämatogene Verbreitung im Körper über infizierte Monozyten und Lymphozyten erreicht das Virus das Gefäßsystem des graviden Uterus oder des zentralen Nervensystems. Die dortige Infektion der Endothelzellen führt zu Mikrovaskulationsstörungen mit Thrombenbildung und Hypoxie (12). Diese immunpathologischen Prozesse verursachen im zentralen Nervensystem Schäden an den Neuronen.

Im Uterus kommt es zu einer vorzeitigen Plazentalösung mit konsekutivem Abort. Außerdem kann das Virus bei geschädigter Plazentaschranke über infizierte Leukozyten den immunologisch ungeschützten Fetus direkt infizieren. Erfolgt die Infektion in einem späten Trächtigkeitsstadium, ist auch die Geburt lebensschwacher Fohlen mit hochgradigen respiratorischen Symptomen mit infauster Prognose möglich (18). Bei der Sektion eines abortierten Fetus werden unter anderem Ikterus, gelbe Flüssigkeitsansammlungen in Thorax und Abdomen, Lungenödem und multifokale stecknadelkopfgroße Nekroseherde in der Leber beobachtet. Pathognomonisch für eine EHV-Infektion des Fetus, aber selten zu finden, sind kleinere oder größere Fibrineinlagerungen in der Trachea und den Hauptbronchien (50). Bleibt die direkte Infektion des Fetus aus, können falsch negative Ergebnisse bei der Untersuchung der Abortursache auftreten, wenn nicht zusätzlich den Eihäuten anhaftende Plazentareste virologisch untersucht werden (43). Eine im Jahr 2002 durchgeführte Studie zeigte, dass eine Vakzinierung gegen EHV die Abortrate deutlich reduzieren kann (21). Wichtig zur Infektionsprophylaxe sind außerdem Stressvermeidung, Quarantäne neu eingestallter Pferde, Isolation infizierter Tiere und die Verhinderung von Kontakt mit infektiösem Material (42).

\section{Equine virale Arteritis (EVA)}

Das Virus der equinen viralen Arteritis ist weltweit verbreitet und weist auch in der deutschen Pferdepopulation eine nicht geringe Seroprävalenz auf, die beispielsweise im Zuchtgebiet WestfalenLippe im Jahre 2003 bei 27,6\% lag (53). Dennoch treten EVA-bedingte Aborte nur sporadisch auf (24). Die Übertragung des Erregers kann über den Respirationstrakt oder durch den Deckakt erfolgen. Eine besondere Rolle bei der Übertragung spielen latent

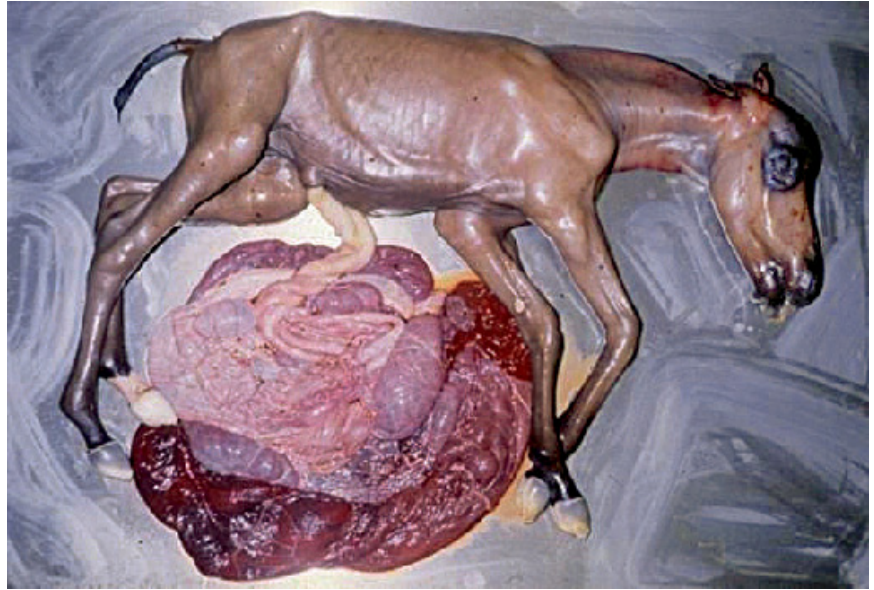

Abb. 1 Abortierter Pferdefetus mit vollständigen Fruchthüllen. Meist kann aus der visuellen Beurteilung nicht auf eine spezifische Abortursache rückgeschlossen werden. Im dargestellten Fall liegt ein Abort durch eine EHVInfektion vor. Typisch ist ein Abortus completus.

Fig. 1 Aborted equine fetus with complete fetal membranes. In most cases the cause of the abortion cannot be found by a gross examination. In the presented case, the abortion results from an EHV infection. An abortus completus is typical.

infizierte Hengste. Sie dienen als natürliches Erregerreservoir und können Stuten sowohl bei der Bedeckung als auch bei der instrumentellen Samenübertragung mit dem Erreger infizieren (46). Die Inkubationszeit beträgt 2-14 Tage. Nach der Infektion infiltriert das Virus die Endothelzellen und Myozyten der Gefäßwände, woraus eine Panvaskulitis resultiert. Bei tragenden Stuten kann die Blutversorgung der Plazenta gestört werden. Dies reduziert sowohl die Sauerstoffversorgung des Fetus als auch die Gestagensynthese. Potenzielle Folgen sind, je nach Zeitpunkt der Infektion, ein Abort oder die Geburt eines lebensschwachen Fohlens (13).

Nach akuter Infektion ist bis zu 3 Wochen eine direkte Ansteckung über Kot, Urin, Speichel, Nasen- und Konjunktivalsekret möglich (1). Die Hauptsymptome einer klinisch manifesten EVAInfektion umfassen Ödeme an den Extremitäten, am Bauch und im Genitalbereich sowie Konjunktivitis, Fieber und gastrointestinale Störungen $(3,25)$. Meist verläuft die Infektion jedoch klinisch inapparent und der Abort ist das einzige Anzeichen (52).

Zum Nachweis eines EVA-bedingten Aborts sollten fetale und plazentare Flüssigkeiten und Gewebe zur Untersuchung mittels Virusanzüchtung und Polymerase-Kettenreaktion (PCR) eingesendet werden. Auch eine zweifache serologische Untersuchung auf Antikörper gegen das EVA-Virus zum Nachweis eines ist als indirekter Erregernachweis möglich. Diese Serumpaaruntersuchungen sollten im Abstand von 2-3 Wochen erfolgen (35).

Hat eine Infektion stattgefunden, kommt es zur Serokonversion (ein Anstieg $\geq 1: 4$ gilt als positiv) und es kann sich ein lebenslanger Infektionsschutz entwickeln. Zur Verhinderung eines Ausbruchs und der Verbreitung dieser meldepflichtigen Erkrankung zählt, neben den allgemeinen Hygienemaßnahmen, vor allem die Identifizierung und der Zuchtausschluss latent infizierter Hengste 
(1). Außerdem steht in Deutschland eine zugelassene inaktivierte Vakzine (Equip Artervac; Zoetis, Berlin) zur Verfügung. Es handelt sich jedoch nicht um einen Markerimpfstoff, wodurch geimpfte Pferde nicht von natürlich infizierten Tieren unterschieden werden können (46). Außerdem führt eine Impfung persistent infizierter Hengste nicht zur Unterdrückung der Virusausscheidung, weshalb die Erhebung des entsprechenden Status der Hengste vor einer Impfung zwingend erfolgen sollte.

\section{Bakteriell bedingte Aborte}

Bakteriell bedingte Aborte treten zum größten Teil im letzten Drittel der Trächtigkeit auf und sind die häufigste Ursache für einen infektiös bedingten Abort (28). Anhand des unterschiedlichen Krankheitsverlaufs und verschiedener morphologischer Veränderungen wird zwischen hämatogener, nokardioformer und aszendierender Plazentitis unterschieden (3).

Die hämatogene Plazentitis wird selten diagnostiziert. Sie ist gekennzeichnet durch diffuse oder multifokale entzündliche Veränderungen des Allantochorions. Auch eine intrauterine Infektion des Fetus kann auftreten. Typische Erreger sind Leptospiren, Chlamydien und Salmonellen (51). Bei der Leptospirose handelt es sich um eine weltweit verbreitete Zoonose. Wichtige Erregerquellen stellen Urin und Kot von Nagetieren dar. Durch das Persistieren der Leptospiren in den Nieren können auch Pferde zu klinisch inapparenten Überträgern werden. Neben einer Allgemeininfektion können Leptospiren bei tragenden Stuten einen Abort auslösen $(18,20)$. Durch den Erreger Salmonella abortusequi induzierte Aborte spielen derzeit keine Rolle (18). Der letzte dokumentierte Ausbruch fand 1997 in Kroatien statt (33). Allerdings können auch andere Salmonellenserovare bei Stuten einen Abort verursachen, weshalb Salmonellen bei der Abklärung eines Abortgeschehens stets berücksichtigt werden sollten. Eine Infektion mit den sich obligat intrazellulär vermehrenden Chlamydien führt zur generalisierten Gefäßinfektion. In der Folge kann es neben einer Infektion von Atemwegen, Gelenken und Augen zu Aborten kommen $(22,47)$.

Über die Pathogenese der sporadisch auftretenden nokardioformen Plazentitis ist bisher wenig bekannt. In der mikrobiologischen Untersuchung werden grampositive, fadenförmige, verzweigte Bakterien nachgewiesen, am häufigsten Amycolatopsis spp. und Crosiella equi. Charakteristisch ist die Infektion der Plazenta im kranialen Bereich des Corpus uteri an der Basis der Uterushörner. Eine Infektion des Fetus erfolgt nicht. Die klinische Diagnose vor dem Auftreten des Aborts ist schwierig, da Symptome wie Ausfluss und vorzeitige Euteranbildung meist fehlen $(6,14)$.

Die Plazentitis durch eine aszendierende Infektion über die Cervix uteri nimmt den größten Stellenwert im Zusammenhang mit dem equinen Abortgeschehen ein. Sie tritt vor allem bei pluriparen Stuten mit anatomischen Defiziten wie mangelndem Vulvaund Hymenalringschluss oder schlechter Zervixkonformation auf (31). Bei den dabei isolierten Keimen spielt Streptococcus equi subspec. zooepidemicus, neben Erregern wie Escherichia coli, Klebsiella pneumoniae und Pseudomonas aeruginosa, die Hauptrolle (19). Durch die aufsteigende Infektion kommt es zur Ödematisierung der zervixnahen Bereiche des Allantochorions. Breitet sich die Plazentitis weiter aus, kann dies die Ablösung der Plazenta vom versorgenden Endometrium zur Folge haben. Zusätzlich kann es zur Infektion des Fetus kommen (3). Ein deutlicher Anstieg der Expression proinflammatorischer Zytokine und Prostaglandine kann Uteruskontraktionen sowie eine Öffnung der Zervix auslösen und so in einem Abort oder einer Frühgeburt resultieren (28). Proinflammatorische Zytokine beschleunigen über die HypothalamusHypophysen-Nebennieren-Achse außerdem die fetale Reifung, sodass die Fohlen auch bei einer Frühgeburt häufig reif genug sind, um zu überleben.

Anders als bei viral bedingten Aborten besteht bei frühzeitiger Diagnose und Therapie die Möglichkeit, einen durch bakterielle Infektionen drohenden Abort zu verhindern. Klassische klinische Symptome einer Plazentitis sind frühzeitige Euteranbildung, mit und ohne Laktation, Vaginalausfluss sowie die Erschlaffung der weichen Geburtswege (49). Viele Plazentitiden verlaufen jedoch subklinisch und erschweren die frühzeitige Diagnosestellung (29). Die Stuten zeigen in der Regel keine Störung des Allgemeinbefindens. Bei Verdacht können wiederholte vergleichende transabdominale und transrektale Ultraschalluntersuchungen zur Diagnosestellung und -sicherung beitragen. Hierbei wird das Augenmerk auf plazentäre Ödeme, Plazentaablösungen, Fruchtwassertrübungen sowie die Herzfrequenz und Aktivität des Fetus gelegt (3). Die transrektale Ultrasonographie kann vor allem bei einer aszendierenden Plazentitis wertvolle Hinweise liefern. Als spezieller Parameter zur Diagnose und Prognoseabschätzung einer Plazentitis dient die sogenannte "combined thickness of uterus and placenta“ (CTUP), gemessen kranioventral der Zervix. Trotz widersprüchlicher Daten über die Aussagekraft der CTUP (30) gelten deutlich über den etablierten Referenzwerten liegende Werte in Kombination mit einer entsprechenden Symptomatik als deutlicher Hinweis für eine Plazentitis $(19,40)$. Zusätzlich wird eine vaginale Untersuchung mittels Spekulum durchgeführt und auf Rötungen, Ausfluss und den Öffnungsgrad der Zervix geachtet (9). Im zervixnahen Bereich sollte eine Tupferprobe zur bakteriologischen Untersuchung, inklusive Antibiogramm, entnommen werden (11). Serologisch kann das Akute-Phase-Protein Serum-Amyloid A, ein sensitiver systemischer Entzündungsmarker beim Pferd, sowohl als prognostischer Indikator als auch zur Überprüfung des Therapieerfolgs herangezogen werden (10). Therapeutisch sollten plazentagängige Breitbandantibiotika (Sulfadimethoxin/Trimethoprim oder eine Kombination aus Penicillin und Gentamicin), nichtsteroidale Antiphlogistika und Altrenogest als synthetisches Gestagen $(0,088 \mathrm{mg} / \mathrm{kg})$ zum Einsatz kommen (20). Bei erfolgtem Abort können makroskopische Veränderungen der Plazenta, wie fokale, multifokale oder diffuse Ödeme und Verfärbungen, Hinweise auf die Erkrankung geben. 


\section{Pilzbedingte Aborte}

Die Häufigkeit eines Aborts durch eine Pilzinfektion liegt Literaturangaben zufolge unter 1\% (24, 27). Dabei spielen die Erreger der Gattungen Aspergillus und Mucor die Hauptrolle. In seltenen Fällen werden Candida spp. als Infektionserreger nachgewiesen $(38,44)$. Wie bei der bakteriell bedingten Plazentitis kommt es meist zur aszendierenden Infektion über den Zervikalkanal mit und ohne Infektion des Fetus. Auch das klinische Bild ähnelt dem einer bakteriell bedingten Plazentitis. Die Behandlung ist schwierig und ein Abort meist nicht zu verhindern.

Als typische makroskopische Veränderungen an der Plazenta treten rundliche Plaques mit nekrotischem Zentrum auf. Zum Nachweis einer Pilzinfektion werden Abstriche von den typischen Läsionen angefärbt und mikroskopisch untersucht. Außerdem können die Pilze mittels kultureller Anzüchtung von Proben aus der Plazenta und der fetalen Lunge und Leber nachgewiesen wer$\operatorname{den}(3)$.

\section{Parasitär bedingte Aborte}

Parasiten gehören zu den seltenen Erregern infektiöser Aborte. Die Infektionserreger Theileria equi und Babesia caballi führen zur equinen Piroplasmose. Da es sich um Blutparasiten handelt, die durch verschiedene Zeckenarten übertragen werden, sind sie vektorenabhängig eher in Südeuropa verbreitet. In Deutschland sind bisher nur Einzelfälle der eqiunen Piroplasmose nachgewiesen. Bedingt durch die globale Erwärmung ist jedoch eine Ausbreitung der Vektoren nach Norden zu verzeichnen und parasitär bedingte Aborte könnten in Zukunft auch in Deutschland einen größeren Stellenwert einnehmen (37).

Klinische Anzeichen einer akuten Piroplasmose sind Fieber, Mattigkeit, Dyspnoe, ikterische Schleimhäute und Ödeme (8). Infektionen mit dem deutlich pathogeneren Parasiten Theileria equi führen in $10-50 \%$ der Fälle zum Tod des betroffenen Tieres. Außerdem kann es zur chronischen Infektion mit unspezifischen Krankheitssymptomen wie Gewichtsverlust und Leistungsmangel kommen (15). Infizierte Tiere bleiben trotz Therapie häufig lebenslange Überträger. Bei einer tragenden Stute kann eine transplazentare Übertragung auf den Fetus erfolgen und einen Abort, eine Totgeburt oder die Geburt eines Fohlens mit neonataler Piroplasmose zur Folge haben. Es besteht aber auch die Möglichkeit, dass ein klinisch gesundes Fohlen geboren wird, das dann als Überträger der Piroplasmose fungiert. Untersuchungen legen nahe, dass mit dem Kolostrum aufgenommene Antikörper eine Parasitämie verhindern können (2). Die Diagnose der Piroplasmose erfolgt über den Nachweis des Erregers im Blut durch Anfärbung (gelingt nur in der Phase der Parasitämie) oder durch PCR. Serologische Tests stehen ebenfalls zur Verfügung.

Encephalitozoon cuniculi, der Erreger der Enzephalitozoonose, tritt vorwiegend beim Kaninchen auf. Auch wenn es keine Fallberichte über die systemische Enzephalitozoonose beim Pferd gibt, können Mikrosporidien seltene Erreger sporadischer Aborte sein, wie in den USA beschrieben wurde (36). Nach einer in Frankreich durchgeführten Studie sollte auch Neospora sp., bekannt als Erreger neurologischer Erkrankungen beim Hund und als Aborterreger beim Rind, im Rahmen eines Abortgeschehens beim Pferd Berücksichtigung finden (38). Informationen über die Bedeutung beim Pferd in Deutschland liegen bisher nicht vor.

\section{Nichtinfektiöse Aborte}

\section{Zwillingsgravidität}

Seit Einführung der Trächtigkeitsuntersuchung mittels Sonographie und den damit verbundenen besseren Möglichkeiten der Diagnostik und Intervention konnte die Häufigkeit der Aborte aufgrund von Zwillingsgraviditäten nach Angaben in der Literatur auf 2,9-6,3\% reduziert werden (24). Im Allgemeinen sind Zwillinge bei Pferden dizygot und somit das Ergebnis einer Doppelovulation, die synchron oder asynchron erfolgen kann. Hinsichtlich des Bedeckungs- oder Besamungsmanagements ist bei Vorhandensein zweier sprungreifer Follikel einerseits eine eventuelle hormonelle Ovulationsinduktion kritisch zu überdenken, andererseits das postovulatorische Monitoring sicherzustellen. Letztgenanntes bezieht sich auf die Ovulationskontrolle sowie die frühzeitige sonographische Trächtigkeitsuntersuchung zur Detektion einer möglichen Zwillingsgravidität, aus der Maßnahmen zur Erzielung einer Einlingsgravidität resultieren.

Je nach Lage der Embryonen wird zwischen uni- und bilateraler Zwillingsgravidität unterschieden. Bei 70\% aller Zwillingsgraviditäten befinden sich beide Fruchtanlagen in einem Uterushorn. Aufgrund des direkten Kontakts der Vesikel und der damit verbundenen verminderten Kontaktfläche zum Endometrium ergibt sich daraus in $85 \%$ der Fälle eine natürliche Selbstreduktion zur Einlingsgravidität bis zum etwa 40. Trächtigkeitstag. Bei bilateralen Zwillingsgraviditäten überleben meist beide Fruchtanlagen die Implantation. Mit zunehmendem Wachstum der Feten kommt es jedoch zum einen zur Versorgungskonkurrenz, zum anderen zur Überdehnung des Uterus. Die Folgen können ein Abort, die Totgeburt oder die Geburt zweier lebensschwacher Fohlen sein. In seltenen Fällen kommt es zum Absterben und zur Mumifikation einer Frucht, wobei sich die zweite Frucht normal weiterentwickelt (3). In einer Studie zur bilateralen Zwillingsgravidität bei Ponystuten wurden in nur 7\% lebende Zwillinge geboren (16). Zur Verhinderung von Aborten durch eine Zwillingsgravidität sind, in Abhängigkeit vom Trächtigkeitsstadium und von der Lokalisation der Fruchtanlagen, verschiedene Reduktionsmöglichkeiten beschrieben (26). Dabei wird die Zwillingsgravidität in eine Einlingsgravidität überführt und dadurch die Abortgefahr reduziert.

\section{Nabelstranganomalien}

Die durchschnittliche Länge der Nabelschnur beim equinen Fetus liegt zwischen 40 und $90 \mathrm{~cm}$. Die starke fetale Mobilität zwischen dem 2. und 7. Trächtigkeitsmonat führt regelmäßig zu leichten Nabelstrangtorsionen ohne klinische Folgen. Kommt es jedoch zu 


\begin{tabular}{|c|c|c|c|}
\hline \multicolumn{4}{|c|}{ Ursache bei $65(52,8 \%)$ von 123 untersuchten equinen Aborten } \\
\hline infektiös & $26,8 \%$ & nichtinfektiös & $26,0 \%$ \\
\hline - bakteriell & $17,1 \%$ & - Zwillingsgravidität & $21,1 \%$ \\
\hline - EHV $1 / 4$ & $8,9 \%$ & - fetale Missbildung & $3,3 \%$ \\
\hline \multirow[t]{2}{*}{ - mykotisch } & $0,8 \%$ & - Nabelstrangulation & $0,8 \%$ \\
\hline & & - Uterustorsion & $0,8 \%$ \\
\hline
\end{tabular}

Tab. 2

Nachgewiesene Abortursachen im Vollblutzuchtgebiet Deutschland Mitte aus den Jahren 1999-2017 ( $n=123$ untersuchte Feten)

Table 2

Causes of abortion in thoroughbred breeding in central Germany between 1999 and 2017 ( $n=123$ examined fetuses). starken Verdrehungen der Nabelschnur und damit einhergehenden hämodynamischen Störungen, kann ein Abort die Folge sein. Bei der pathologischen Untersuchung können Ödeme, Farbveränderungen, Thrombosen und Aufweitungen der Nabelschnurgefäße im Zusammenhang mit einer Nabelschnurtorsion den Verdacht bestätigen $(34,51)$. Eine sehr lange Nabelschnur kann außerdem zum Absterben des Fetus durch Strangulation führen (3).

In seltenen Fällen lösen neoplastische Veränderungen an der Nabelschnur einen Abort aus (4).

\section{Eihautwassersucht}

Die normale Fruchtwassermenge im späten Trächtigkeitsstadium beträgt zwischen 11 und 20 Litern (23). Eine pathologischen $\mathrm{Zu}$ nahme der Fruchtwässer wird als Eihautwassersucht bezeichnet. Diese tritt meist zwischen dem 8. und 10. Trächtigkeitsmonat auf und betrifft vor allem pluripare Stuten (50). Abhängig von der Lokalisation wird zwischen Hydrallantois und Hydramnion unterschieden, wobei auch Mischformen möglich sind. Als Hauptsymptom tritt eine deutliche Umfangsvermehrung des Abdomens auf, die sich innerhalb weniger Tage bis Wochen entwickelt. Mögliche Folgen sind Kolik, erhöhte Atem- und Herzfrequenz, verminderter Appetit, gestörtes Allgemeinbefinden und ein Absterben des Fetus. Rektal lässt sich der stark dilatierte, meist nach dorsal ausgeweitete Uterus unmittelbar nach Überwinden des Anus palpieren. Aufgrund der großen Flüssigkeitsmengen im Uterus sind häufig keine Fruchtteile tastbar und die Die Gebärmutterwand ist in der Regel gespannt (9).

Bei der Pathogenese der Eihautwassersucht spielen Plazentadysfunktionen eine wichtige Rolle. $\mathrm{Zu}$ weiteren potenziellen Ursachen gehören Plazentitis, fetale Missbildungen und Zwillingsgravidität. Es handelt sich jedoch nur um Hypothesen zur Pathogenese. Ist das Allgemeinbefinden der Stute gestört, besteht die Therapie in einer Abortinduktion mittels Oxytozin (17).

\section{Wollraupenspinner}

In den USA und Australien stellten Raupen (Eastern tent caterpillar bzw. processionary caterpillar) eine bedeutende Ursache umweltbedingter Aborte dar. In den USA wurde diese Abortursache 2001 als „mare reproductive loss syndrome" (MRLS) beschrieben. Die metaphylaktische Maßnahme der Entfernung der bevorzugten Wirtsbäume (Schwarzkirsche) der Raupen auf Pferdehaltungs- flächen minimierte die Erkrankungsinzidenz. Ein ähnliches Abortgeschehen wurde 2004 in Australien beobachtet und mit dem Begriff „equine amnionitis and fetal loss" (EAFL) beschrieben. Die genaue Pathogenese beider Erkrankungen ist nicht abschließend geklärt. In mehreren experimentellen Untersuchungen ließ sich jedoch zeigen, dass die orale Aufnahme der Raupenhaare einen Abort auslösen kann. Nach Invasion in das Gefäßsystem werden die Haarfragmente im Körper verteilt und erreichen so auch den Uterus und Fetus. In der Folge kommt es zu einer lokalen Entzündung mit Tod des Fetus und Abort. Man geht davon aus, dass die Haarfragmente als Vektoren für Bakterien dienen. Charakteristisch sind entzündliche Veränderungen im Bereich des Amnions und des Nabelstrangs $(7,48)$.

\section{Eigene Untersuchung}

Das Ziel dieser retrospektiven Studie war es, einen Überblick über die Bedeutung verschiedener Abortursachen in Deutschland zu erhalten und diese mit den Angaben in der Literatur zu vergleichen. Dazu wurden Abortfälle aus den Jahren 1999 bis 2017 im Vollblutzuchtgebiet Deutschland Mitte (Hessen, Saarland, Rheinland-Pfalz, Baden-Württemberg) ausgewertet. Die Analyse umfasste 123 Abortfälle. Bei allen abortierten Früchten erfolgten im Institut für Veterinär-Pathologie der Justus-Liebig-Universität Gießen eine pathologisch-anatomische Untersuchung sowie eine histopathologische Untersuchung von Thymus, Herz, Knochenmark, Lunge, Milz, Leber, Nieren, Nabelstrang, Plazenta, Magen, Darm und ZNS. Des Weiteren wurden bakteriologische und mykologische Untersuchungen von Organproben sowie eine PCR-Untersuchung auf EHV1/4-spezifische Gensequenzen am Institut für Hygiene und Infektionskrankheiten der Tiere der Justus-Liebig-Universität Gießen vorgenommen. Diese Untersuchungen führten in 65 Fällen $(52,8 \%$ ) zur Klärung der Abortursache ( $\$$ Tab. 2).

Bei den bakteriell bedingten Aborten handelte es sich überwiegend um Infektionen mit Streptococcus equi subsp. zooepidemicus (48\%), gefolgt von Infektionen mit E. coli (28\%) und Klebsiella pneumoniae (14\%). Vereinzelt wurden Enterococcus casseliflavus (5\%) und Actinobacillus equuli (5\%) als Erreger nachgewiesen. Die Pilzspezies des Aborts mykotischen Ursprungs konnte nicht ermittelt werden.

In $47,2 \%$ der Fälle war keine eindeutige Abortursache eruierbar. Im nationalen Vergleich liegt die Rate in der Größenordnung, 
die von Hörügel und Pöhle (24) mit $42 \%$ genannt wird. Für die Praxis ist daher festzuhalten, dass sich mit den derzeitigen Untersuchungsmethoden bei $40-50 \%$ der Aborte keine Ursache nachweisen lässt.

Die Ursache für die hohe Anzahl von Fällen ohne nachgewiesene Ursache könnte in autolytischen Prozessen einzelner Organe liegen. Außerdem ist der Nachweis einiger seltenerer abortigener Erreger schwierig und fehlt in der routinemäßigen Diagnostik (z. B. EVA-Virus, Chlamydien, Salmonellen). Hinzu kommt, dass die Abortursache auch bei der Mutterstute liegen kann (39) und in diesen Fällen keine verwertbaren Befunde im Fetus oder den Fruchthüllen zu finden sind. Dies gilt beispielsweise für endokrine Störungen in der Trächtigkeit.

Der prozentuale Anteil infektiöser Aborte in der deutschen Vollblutzucht im Auswertungszeitraum ist im Vergleich dem in den Jahren 1996-1999 (25,5\%) annähernd gleich geblieben (33). Untersuchungen aus Sachsen (Jahre 2002-2007) zeigen hingegen mit $38 \%$ einen deutlich höheren Anteil infektiöser Aborte (24). Die Ursache für diese Diskrepanz ist unklar, da davon auszugehen ist, dass Vollblutstuten in Deutschland dem gleichen Erregerdruck ausgesetzt sind wie Pferde anderer Rassen.

Erfreulich ist der Rückgang der zwillingsbedingten Aborte um $19 \%$, verglichen mit dem Resultat der Studie aus der Deutschen Vollblutzucht zwischen 1969 und 1999 (33). Mögliche Erklärungen bestehen in den verbesserten diagnostischen Möglichkeiten mittels Ultrasonographie und den umfassenderen therapeutischen Optionen der Zwillingsreduktion in der heutigen Zeit. Nichtdestotrotz zeigen die Daten der eigenen Untersuchung, dass Zwillingsgraviditäten nach wie vor die häufigste nachgewiesene nichtinfektiöse Abortursache darstellen.

\section{Schlussfolgerung}

Sowohl die Literaturübersicht als auch die eigenen Auswertungsergebnisse belegen, dass das im Tierartenvergleich grundsätzlich hohe Abortrisiko für Stuten (bedingt durch die Vielzahl potenziell abortauslösender Ursachen) nach wie vor besteht. Auch wenn etwa die Hälfte aller Aborte retrospektiv nicht einer singulären auslösenden Noxe zuzuordnen und somit kausal aufzuklären ist, sollten seitens der Züchter und betreuenden Tierärzte alle Bestrebungen hierzu unternommen und Abortmaterial grundsätzlich gesichert und der eingehenden Untersuchung zugeführt werden. Die Zusammenstellung der Literatur zeigt, dass durchaus mehr Infektionserreger einen Abort beim Pferd verursachen können, als in der Routineuntersuchung erfasst werden. Es ist anzuregen, dass in den Diagnostiklaboren die Anzahl der erfassten Erreger erhöht wird, um die Rate aufgeklärter Abortfälle zu steigern.

Bezüglich der nicht infektiös bedingten Aborte, hier insbesondere im Hinblick auf Zwillingsgravidität/Zwillingsabort, kann das Risiko durch prophylaktische (Besamungsmanagement bei drohender Doppelovulation), metaphylaktische (sonographischer Ausschluss einer Zwillingsgravidität) oder therapeutische Maß- nahmen (Reduktion einer Zwillings- zur Einlingsgravidität) erheblich minimiert werden, wie die Ergebnisse aus der deutschen Vollblutzucht eindrücklich belegen.

Im Fall der infektiös bedingten Aborte gelten, aufgrund der Diversität der potenziellen Noxen bei gleichzeitig häufig problematischem Nachweis des verursachenden Erregers sowie des regelmäßig hohen Übertragungsrisikos auf noch nicht infizierte tragende Stuten, nach wie vor die Grundsätze des professionellen Abortmanagements: Jeder aufgetretene Abort muss der Untersuchung zum Versuch der kausalen Aufklärung zugeführt werden und ist grundsätzlich zunächst als infektiös bedingt zu bewerten, bis das Gegenteil bewiesen ist. Als wichtigste Maßnahme gilt die Vermeidung der Weiterverbreitung einer potenziellen Infektion.

\section{Interessenkonflikt}

Die Autoren bestätigen, dass kein Interessenkonflikt besteht.

\section{Literatur}

1. Ahlswede L, Zurmühlen K. Was sollte der praktische Tierarzt über die EVA (Equine Virus Arteritis) wissen? Prakt Tierarzt 2001; 82: 1040-1044.

2. Allsoppa MTEP, Lewisb BD, Penzhornb BL. Molecular evidence for transplacental transmission of Theileria equi from carrier mares to their apparently healthy foals. Vet Parasitol 2007; 148: 130-136.

3. Aurich C. Fetale Phase. In: Reproduktionsmedizin beim Pferd. Aurich C, Hrsg. Stuttgart: Parey 2009; 158-174.

4. Binanti D, Livini M, Riccaboni P, Sironi G. A case of umbilical cord teratoma in an aborted foal. J Vet Diagn Invest 2013; 25: 173-175.

5. Bollwein H. Störungen der Trächtigkeit. In: Reproduktionsmedizin beim Pferd. Aurich C, Hrsg. Stuttgart: Parey 2005: 155-172.

6. Canisso IF, Ball BA, Erol E, Claes A, Scoggin KE, McDowell KJ, Williams NM, Dorton AR, Wolfsdorf KE, Squires EL, Troedsson MH. Attempts to induce nocardioform placentitis (Crossiela equi) experimentally in mares. Equine Vet J 2015; 47: 91-95.

7. Cawdell-Smith AJ, Todhunter KH, Anderson ST, Perkins NR, Bryden WL. Equine amnionitis and fetal loss: Mare abortion following experimental exposure to Processionary caterpillars (Ochrogaster lunifer). Equine Vet J 2012; 44: 282-288.

8. Chhabra S, Uppal SK, Ranjan R, D. SL. Transplacental transmission of Babesia equi (Theileria equi) from carrier mares to foals. J Parasit Dis 2012; 36: 31-33.

9. Clausen P-H, Ennen S, Handler J, Hospes R, Scharner D, Sieme H, Wehrend A. Reproduktionsstörungen. In: Handbuch Pferdepraxis. Brehm W, Gehlen H, Ohnesorge B, Wehrend A, Hrsg. Stuttgart: Enke 2017; 628-699.

10. Coutinho da Silva MA, Canisso IF, MacPherson ML, Johnson AE, Divers TJ. Serum amyloid A concentration in healthy periparturient mares and mares with ascending placentitis. Equine Vet J 2013; 45: 619-624.

11. Cummins C, Carrington S, Fitzpatrick ED, Duggan V. Ascending placentitis in the mare: A review. Irish Vet J 2008; 61: 307-313.

12. Damiani AM, de Vries M, Reimers G, Winkler S, Osterrieder N. A severe equine herpesvirus type 1 (EHV-1) abortion outbreak caused by a neuropathogenic strain at a breeding farm in northern Germany. Vet Microbiol 2014; 172: 555-562.

13. Del Piero F. Equine Viral Arteritis. Vet Pathol 2000; 37: 287-296.

14. Erol E, Sells SF, Williams NM, Kennedy L, Locke SJ, Labeda DP, Donahue JM, Carter CN. An investigation of a recent outbreak of nocardioform placentitis caused abortions in horses. Vet Microbiol 2012; 158: 425-430.

15. Feige K, Fey K. Krankheiten des hämatopoetischen Systems. In: Handbuch Pferdepraxis. Brehm W, Gehlen H, Ohnesorge B, Wehrend A, Hrsg. Stuttgart: Enke 2017; 284-320. 
16. Ginther OJ, Griffin PG. Natural outcome and ultrasonic identification of equine fetal twins. Theriogenology 1994; 41: 1193-1199.

17. Govaere JLJ, De Schauwer C, Hoogewijs MK, Chiers K, Lefere L, Catry B, Roels K, van Heerden M, Broeckx S, de Kruif A. Hydrallantois in the mare - a report of five cases. Reprod Domest Anim 2013; 48: 1-6.

18. Haas L, Osterrieder K, Selbitz H-J, Valentin-Weigand P, von Samson-Himmelstjerna G. Inkektionskrankheiten. In: Handbuch Pferdepraxis. Brehm W, Gehlen H, Ohnesorge B, Wehrend A, Hrsg. Stuttgart: Enke 2017; $1108-1135$.

19. Hahn K, Witte TS. Die Plazentitis der Stute. Prakt Tierarzt 2016; 97: 998-1008.

20. Hamond C, Martins G, Bremont S, Medeiros MA, Bourhy P, Lilenbaum W. Molecular Characterization and Serology of Leptospira kirschneri (Serogroup Grippotyphosa) Isolated from Urine of a Mare Post-Abortion in Brazil. Zoonoses and Public Health 2016; 63: 191-195.

21. Heldens JG. Wirksamkeitsnachweis einer inaktivierten EHV1, 4 VollvirusVakzine im Challengeversuch an Fohlen und tragenden Stuten. Prakt Tierarzt 2002; 83: 348.

22. Henning K, Sachse K, Sting R. Demonstration of Chlamydia from an equine abortion. Dtsch Tierärztl Wochenschr 2000; 107: 49-52.

23. Hoffmann B, Leiser R, Schuler G. Physiologie und Anatomie der Trächtigkeit, Trächtigkeitsdiagnostik. In: Reproduktionsmedizin beim Pferd. Aurich C, Hrsg. Stuttgart: Parey 2009; 125-154.

24. Hörügel U, Pöhle D. Abortursachen bei Pferden in Sachsen von 2002-2007. Prakt Tierarzt 2008; 89: 644-647.

25. Hörügel U, Dietz U, Muluneh A, Hardt M, Wensch HJ, May M. Auftreten der Equinen Virusarteritis in zwei sächsischen Pferdebeständen - ein Fallbericht. Prakt Tierarzt 2008; 89: 484-491.

26. Klewitz J, Heberling A, Ortgies F, Sieme H. Erfolgsraten nach transvaginalultraschallgeleiteter Fruchtwasseraspiration und fetaler Punktion bei der Stute. Pferdeheilkunde 2010; 26: 797-803.

27. Laugier C, Foucher N, Sevin C, Leon A, Tapprest J. A 24-year retrospective study of equine abortion in Normandy (France). J Equine Vet Sci 2011; 31: 116-123.

28. LeBlanc MM, Giguere S, Brauer K, Paccamonti DL, Horohov DW, Lester GD, O'Donnell LJ, Sheerin BR, Pablo L, Rodgerson DH. Premature delivery in ascending placentitis is associated with increased expression of placental cytokines and allantoic fluid prostaglandins E2 and F2a. Theriogenology 2002; 58: 841-844.

29. LeBlanc MM. Ascending placentitis in the mare: an update. Reprod Domest Anim 2010; 45 Suppl 2: 28-34.

30. Löf HK, Gregory JW, Neves AP, Jobim MIM, Gregory RM, Mattos RC. Combined thickness of the uterus and placenta (CTUP) as indicator of placentitis in Thoroughbred mares. Pferdeheilkunde 2014; 30: 37-41.

31. Macpherson ML, Bailey CS. A clinical approach to managing the mare with placentitis. Theriogenology 2008; 70: 435-440.

32. Madic J, Hajsig D, Sostaric B, Curic S, Soel B, Naglic T, Cvetnic Z. An outbreak of abortion in mares associated with Salmonella abortus equi infection. Equine Vet J 1997; 29: 230-233.

33. Merkt H, Klug E. Vergleich der Fohlenverluste in der Deutschen Vollblutzucht über drei Jahrzehnte. Pferdeheilkunde 2001; 17: 203-207.

34. Mizushima C. Late-term abortion associated with umbilical cord torsion in the mare: Case report. J Equine Vet Sci 2005; 25: 162-163.

35. Mohr C. Untersuchungen zur Immunisierung gegen die Equine Virale Arteritis. Dissertation, Veterinärmedizinische Fakultät der Universität Leipzig 2006.
36. Patterson-Kane JC, Caplazi P, Rurangirwa F, Tramontin RR, Wolfsdorf K. Encephalitozoon cuniculi placentitis and abortion in a Quarterhorse mare. J Vet Diagn Invest 2003; 15: 57-59.

37. Pikalo J, Sattler T, Eichinger M, Loitsch A, Schmoll F, Schusser GF. Vorkommen von Antikörpern gegen Babesia caballi und Theileria equi bei Pferden in Mitteldeutschland. Pferdeheilkunde 2016; 32: 254-259.

38. Pitel P, Romand S, Pronost S, Foucher N, Gargala G, Maillard K, Thulliez P, Collobert-Laugier C, Tainturier D, Fortier G, Ballet JJ. Investigation of Neospora sp. antibodies in aborted mares from Normandy, France. Vet Parasitol 2003; 118: 1-6.

39. Pospischil A, Lieb A, Corboz L. Ursachen pränataler Fohlenverluste in der Schweiz. Schweiz Arch Tierheilk 1992; 143: 401-409.

40. Renaudin CD, Troedsson MH, Gillis CL, King VL, Bodena A. Ultrasonographic evaluation of the equine placenta by transrectal and transabdominal approach in the normal pregnant mare. Theriogenology 1997; 47: 559-573.

41. Ricketts SW, Barrelet A, Whitwell KE. A review of the causes of abortion in UK mares and means of diagnosis used in an equine studfarm practice in Newmarket. Pferdeheilkunde 2001; 17: 589-592.

42. Scheepers K, Müller H. Fertilitätsrelevante Virusinfektionen beim Pferd. Pferdeheilkunde 1997; 13: 445-449.

43. Smith KC, Whitwell KE, Blunden AS, Bestbier ME, Scase TJ, Geraghty RJ, Nugent J, Davis-Poynter NJ, Cardwell JM. Equine herpesvirus-1 abortion: atypical cases with lesions largely or wholly restricted to the placenta. Equine Vet J 2004; 36: 79-82.

44. Stefanetti V, Marenzoni ML, Lepri E, Coletti M, Proietti PC, Agnetti F, Crotti S, Pitzurra L, Del Sero A, Passamonti F. A case of Candida guilliermondii abortion in an Arab mare. Medical Mycology Case Reports 2014; 4: $19-22$.

45. Thein P, Eßich G, Röhm A. Fohlenerkrankungen und Fohlenverluste - ein Beitrag zur Ursache von Aborten im Zeitraum von 1972 bis 2002 im Haupt- und Landgestüt Marbach an der Lauter. Tierärztl Umsch 2005; 60: 115-127.

46. Thein P. Zur Arteritisvirus-Infektion des Pferdes: Aktuelle Probleme. Prakt Tierarzt 2007; 88: 896-901.

47. Thein P. Infektiöse Aborte der Stuten - Ätiologie, Immunpräventive und Bekämpfung. Pferdeheilkunde 2012; 28: 171-186.

48. Todhunter KH, Cawdell-Smith AJ, Bryden WL, Perkins NR, Begg AP. Processionary Caterpillar Setae and Equine Fetal Loss: 1. Histopathology of Experimentally Exposed Pregnant Mares. Vet Pathol 2014; 51: 1117-1130.

49. Troedsson MHT, Miller LMJ. Equine placentitis. Pferdeheilkunde 2016; 32: 49-53.

50. Von Oppen T, Bartmann C-P. Two cases of hydrallantois in the mare. Pferdeheilkunde 2001; 17: 593-596.

51. Whitwell KE. Abortions and stillbirths: A pathologist's overview. In: Equine Reproduction, 2nd edn. McKinnon AO, Squires EL, Vaala WE, Varner DD, eds. Hoboken, NJ, USA: John Wiley \& Sons 2011; 2339-2349.

52. Williams NM. Disorders of Horses. In: Kirkbrides Diagnosis of Abortion and Neonatal Loss in Animals. Njaa B, Kirkbride CA, eds. Chichester, West Sussex: Wiley-Blackwell 2012; 147-171.

53. Zurmühlen K, Ahlswede L, Baljer G, Herbst W. Seroepidemiologische Untersuchungen zur Verbreitung des equinen Arteritisvirus (EAV) innerhalb eines Zuchtgebietes. Tierärztl Prax 2003; 31 (G): 281-286. 